\title{
Impedimetric, diamond-based immmunosensor for the detection of C-reactive protein
}

\author{
V. Vermeeren ${ }^{\mathrm{a}}$, L. Grieten ${ }^{\mathrm{b}}$, N. Vanden Bon ${ }^{\mathrm{a}}$, N. Bijnens ${ }^{\mathrm{b}, 1}$, S. Wenmackers ${ }^{\mathrm{b}, 2}$, S.D. Janssens ${ }^{\mathrm{b}}$, \\ K. Haenen ${ }^{\mathrm{b}, \mathrm{c}}$, P. Wagner ${ }^{\mathrm{b}}$, L. Michiels ${ }^{\mathrm{a}, *}$ \\ a Hasselt University and Transnational University Limburg, School for Life Sciences, Biomedical Research Institute, Agoralaan, Bldg. C, B-3590 Diepenbeek, Belgium \\ ${ }^{\mathrm{b}}$ Hasselt University and Transnational University Limburg, School for Life Sciences, Institute for Materials Research, Wetenschapspark 1, B-3590 Diepenbeek, Belgium \\ ${ }^{\mathrm{c}}$ IMEC vzw, Division IMOMEC, Wetenschapspark 1, B-3590 Diepenbeek, Belgium
}

\section{A R T I C L E I N F O}

\section{Article history:}

Received 3 January 2011

Received in revised form 8 March 2011

Accepted 19 March 2011

Available online 25 March 2011

\section{Keywords:}

Cardiovascular diseases

C-reactive protein

Immunosensor

Impedance spectroscopy

Nanocrystalline diamond

Real-time detection

\begin{abstract}
A B S T R A C T
The high prevalence of cardiovascular diseases (CVD) demands a reliable and sensitive risk assessment technique. In order to develop a fast and label-free immunosensor for C-reactive protein (CRP), a risk factor for this condition, anti-CRP antibodies were physically adsorbed to the hydrogen $(\mathrm{H})$-terminated surface of nanocrystalline diamond (NCD). An Enzyme-Linked ImmunoSorbent Assay (ELISA) reference technique showed that this was a suitable substrate for antibody-antigen recognition reactions. Electrochemical Impedance Spectroscopy (EIS) was used to electronically detect CRP recognition. The specificity of the immunosensor was demonstrated by incubation with CRP and plasminogen as reference molecule. A different impedance behavior was observed in real-time after CRP addition as compared to plasminogen addition: the impedance increased only during CRP incubation. Fitting the data showed that this corresponded with a decrease in capacitance of the molecular layer due to its increased thickness by specific CRP recognition. Sensitivity experiments in real-time showed a clear discrimination between $1 \mu \mathrm{M}, 100 \mathrm{nM}$, and $10 \mathrm{nM}$ of CRP after $10 \mathrm{~min}$ at $100 \mathrm{~Hz}$. Since, $10 \mathrm{nM}$ of CRP was still clearly distinguishable from buffer solution, our CRP-directed immunosensor prototype reaches a sensitivity that is within the physiologically relevant concentration range of this biomarker in healthy controls and CVD patients. Moreover, this prototype displayed real-time discriminating power between spiked and unspiked serum, and thus also shows its applicability in this biological matrix.
\end{abstract}

(c) 2011 Elsevier B.V. All rights reserved.

\section{Introduction}

C-reactive protein (CRP) is an acute phase protein that is produced in the liver. In its native form, it is a pentamer of $126 \mathrm{kDa}$, consisting of five identical monomers of $25 \mathrm{kDa}$ each. Its serum level increases rapidly during systemic inflammation, where it assists in complement binding to foreign and damaged cells. Through this mechanism, phagocytosis is enhanced by macrophages, which express a receptor for CRP. There is also evidence that CRP increases the expression of the cell adhesion molecules ICAM-1, VCAM- 1 and E-selection in cultured endothelial cells [1]. Therefore, CRP has traditionally been used as a reliable marker for tissue injury, infection, and inflammation. However, studies have shown that CRP is also

\footnotetext{
* Corresponding author. Tel.: +32 11 269320; fax: +32 11269239.

E-mail address: luc.michiels@uhasselt.be (L. Michiels).

1 Current address: Eindhoven University of Technology, Den Dolech 2, 5600 MB Eindhoven, The Netherlands.

2 Current address: Rijksuniversiteit Groningen, Broerstraat 5, 9712 CP Groningen, The Netherlands.
}

implicated in the development of cardiovascular diseases (CVD), such as atherosclerosis, angina, coronary heart disease, peripheral artery disease, myocardial infarction, and stroke [2]. Not only can elevated blood levels of CRP predict the recurrence of coronary events in patients with stable or unstable angina [2], but also it can even determine an increased risk for hypertension [3] and CVD in healthy controls [4]. CRP serum levels of $<8 \mathrm{nM}$ are considered normal, while levels $>25 \mathrm{nM}$ indicate an increased risk for CVD. As a result, CRP can serve as an important risk assessment factor for the development of cardiovascular diseases [4].

The most recent statistics from the American Heart Association report that in 2006, 80 million Americans suffered from CVD, of whom 829,072 actually died [5]. It is estimated that 2400 Americans die of CVD each day [6]. Hence, the need for reliable and sensitive risk assessment is obvious. Over the years, many assay methods for the detection of CRP have been developed and improved, such as latex agglutination, nephelometry, turbidimetry, and fluorescent polarization [7]. Currently, an Enzyme-Linked ImmunoSorbent Assay (ELISA)-based blood test called the 'highly sensitive C-reactive protein (hs-CRP) assay' is available to determine CVD risk (Dade Behring, Inc., now Siemens Medical, IL, 
USA). Its limit of detection is $0.8 \mathrm{nM}\left(0.1 \mu \mathrm{g} \mathrm{ml} \mathrm{m}^{-1}\right)$. However, disadvantages of ELISA tests are the long reaction times (several hours) of the multiple steps involved, and the requirement of trained personnel and expensive reagents [8]. A Surface Plasmon Resonance (SPR) biosensing platform for CRP was developed by $\mathrm{Hu}$ et al., where anti-CRP antibodies were bound to a gold (Au) surface through Protein G. They reached a detection limit of $8 \mathrm{nM}\left(1 \mu \mathrm{g} \mathrm{ml}^{-1}\right)$ of purified CRP [8]. Meyer et al. also established an immunosensor for CRP detection based on SPR. The detection principle was based on two different anti-CRP antibodies: one for CRP entrapment and one for labeling. A linear detection range of $16-40 \mathrm{nM}\left(2-5 \mu \mathrm{g} \mathrm{ml}^{-1}\right)$ CRP in PBS buffer was found [9]. Recently, the same authors developed a novel CRP detection method in crude serum, urine, and saliva samples based on magnetic detection. With its linear detection range from $0.2 \mathrm{nM}\left(25 \mathrm{ng} \mathrm{ml}^{-1}\right)$ to $20 \mathrm{nM}\left(2.5 \mu \mathrm{g} \mathrm{ml}^{-1}\right)$, it showed to be even more sensitive than the hs-CRP ELISA assay [10]. In the last years, the focus on Electrochemical Impedance Spectroscopy (EIS)-based immunosensing methods for various targets has increased significantly. These impedimetric immunosensors are usually based on capacitive changes or changes based on charge-transfer resistance. Cooreman et al. investigated the interaction between the semiconducting polymer poly[2-methoxy-5(3,7-dimethyloctyloxy)]-1,4-phenylenevinylene (MDMO-PPV) and physically adsorbed antibodies against the fluorescent dyes FITC and Cy5. Specific antigen-antibody recognition was demonstrated based on EIS. These antibody-modified polymer films show a clear response to $9 \mathrm{nM}$ of antigen solution, with a time constant of 2-3 min [11]. Ionescu et al. devised an impedimetric Au-based immunosensor against the antibiotic ciprofloxacin. A detection limit of $80 \mathrm{fM}\left(10 \mathrm{pg} \mathrm{ml}^{-1}\right)$ was reached [12]. Yang et al. used EIS to directly detect antigen-antibody binding. They covalently bound human IgG and IgM, serving as antigens, to aldehyde-terminated nanocrystalline diamond (NCD) surfaces using a glyceraldehyde crosslinker. The antibodies were detected in real-time using antiIgG and anti-IgM [13]. In this study, we will adsorb monoclonal anti-CRP antibodies onto hydrophobic H-terminated NCD, allowing CRP recognition to occur through a typical antigen-antibody interaction via the hypervariable regions of anti-CRP, adding clinical relevance to our immunosensor setup in comparison with the work of Yang et al. [13].

Diamond has been studied as a novel transducer material for biosensor fabrication because of very appealing physical, optical, chemical and electrical characteristics. For a complete overview, the reader is referred to the works of Field and Swain $[14,15]$. Among others, it has a wide electrochemical window, and, although an insulator with a very wide band gap of $5.5 \mathrm{eV}$, it can be made electrically conductive by chemical doping [16-18]. These electrical properties of diamond have already been exploited in the construction of diamond-based field-effect transistors (FET) using technologies that are compatible with lithographic methods [19,20]. Moreover, diamond is biocompatible, allowing in vivo applications [21] and it can be functionalized with biomolecules. Although diamond is chemically inert, Takahashi et al. surmounted this barrier by the photochemical activation of $\mathrm{H}$-terminated diamond surfaces [22]. The way was paved towards further modification of diamond with biomolecules, such as DNA and proteins. Nowadays, also wet chemical [23], electrochemical [24-26], and photochemical [25-30] methods exist to functionalize diamond surfaces. The reader is referred to the article of Wenmackers for an extensive review on functionalization and read-out techniques of DNA on diamond [31]. In our previous work, it was demonstrated that real-time differentiation between fully complementary and 1-mismatch DNA was possible on lightly boron (B)-doped nanocrystalline diamond (NCD) substrates using EIS [32]. In order to achieve a more generic, flexible biosensor platform, this same
NCD- and EIS-based setup was developed in an immunosensing application.

\section{Materials and methods}

\subsection{Materials}

Sulfuric acid $\left(\mathrm{H}_{2} \mathrm{SO}_{4}\right)$ was obtained from VWR International (Zaventem, Belgium). Potassium nitrate $\left(\mathrm{KNO}_{3}\right)$ was acquired from Merck (Overijse, Belgium). Coating buffer (0.014 $\mathrm{M} \mathrm{Na}_{2} \mathrm{CO}_{3}$, $\left.0.035 \mathrm{M} \mathrm{NaHCO}_{3}, 0.003 \mathrm{M} \mathrm{NaN}_{3}\right)$, Phosphate Buffered Saline (10× PBS: $1.29 \mathrm{M} \mathrm{NaCl}, 0.05 \mathrm{M} \mathrm{Na}_{2} \mathrm{HPO}_{4} \cdot 2 \mathrm{H}_{2} \mathrm{O}, 0.015 \mathrm{M} \mathrm{KH}_{2} \mathrm{PO}_{4}, \mathrm{pH}$ 7.2 ) and CRP buffer (20 mM TRIS-buffer, pH 8, containing $0.28 \mathrm{M}$ $\mathrm{NaCl}, 0.09 \% \mathrm{NaN}_{3}$ and $5 \mathrm{mM} \mathrm{CaCl}_{2}$ ) were homemade. Bovine Serum Albumin (BSA) was bought from Roche Diagnostics (Vilvoorde, Belgium). An ELISA amplification system based on the cyclic redox reaction of $\mathrm{NADH} / \mathrm{NAD}^{+}$was obtained from Invitrogen (Merelbeke, Belgium). Unlabeled and alkaline phosphatase (AP)-labeled anti-CRP monoclonal antibodies, their specific antigen CRP, and plasminogen for the specificity experiments were synthesized by Scipac (Kent, United Kingdom). Human serum is obtained by centrifuging whole blood from a healthy donor at $4000 \mathrm{rpm}$ for $15 \mathrm{~min}$ at $5{ }^{\circ} \mathrm{C}$. Quantification of the CRP levels was performed in Labo Rigo (Genk, Belgium) by a turbidimetric assay, which indicated a CRP concentration well below $4 \mathrm{nM}$.

\subsection{NCD synthesis and functionalization}

Lightly B-doped H-terminated NCD was synthesized by microwave plasma enhanced chemical vapor deposition in a way previously described $[28,32,33]$. Also, commercial NCD samples from rho-BeSt Coatings (Innsbruck, Austria, order no. HASTPO43) were used. The comparability of biosensing experiments on IMO NCD samples and commercially available H-terminated NCD samples from rho-BeSt was already demonstrated in previous studies $[28,32]$. Thus, these two types of samples were used interchangeably.

Unlabeled anti-CRP monoclonal capture antibodies were diluted in coating buffer until a concentration of $20 \mathrm{nM}$ was reached. The H-terminated NCD samples were immersed in this anti-CRP solution and incubated for $2 \mathrm{~h}$ at $37^{\circ} \mathrm{C}$ to allow physisorption of the antibodies. The anti-CRP-coated NCD samples were subsequently incubated overnight in a $6 \%$ BSA solution in $1 \times \mathrm{PBS}$ at $4{ }^{\circ} \mathrm{C}$ to block the remainder of the NCD surface that was not coated with anti-CRP.

Also, H-terminated NCD samples treated only with 6\% BSA but without anti-CRP antibodies were used as a control.

\subsection{Reference ELISA procedure to verify the functionality of the adsorbed antibodies}

To verify the formation of the anti-CRP/CRP-complex, $20 \mathrm{nM}$ of unlabeled anti-CRP capture antibodies and 6\% BSA were coated on two H-terminated NCD samples using the method described in Section 2.2. One NCD sample was subsequently incubated with $2.4 \mu \mathrm{M}$ of CRP, while the other was treated with CRP buffer without CRP. After $2 \mathrm{~h}$ of reaction, detection occurred through the incubation with $70 \mathrm{mM}$ AP-labeled anti-CRP antibodies in conjugation buffer for $1 \mathrm{~h}$, and a subsequent substrate reaction using an ELISA amplification system based on NADPH. This substance is transformed by AP to NADH, which is the substrate for a secondary enzymatic redox cycle. Diaphorase oxidizes NADH to NAD ${ }^{+}$while reducing a tetrazolium salt to an intensely colored formazan dye. Subsequently, while ethanol is oxidized by alcohol dehydrogenase, $\mathrm{NAD}^{+}$is again reduced to $\mathrm{NADH}$, driving the cyclic behavior of the amplification reaction. After 1-2 min, the reaction liquid was removed from the surface of the NCD samples with a micropipette, and transferred 


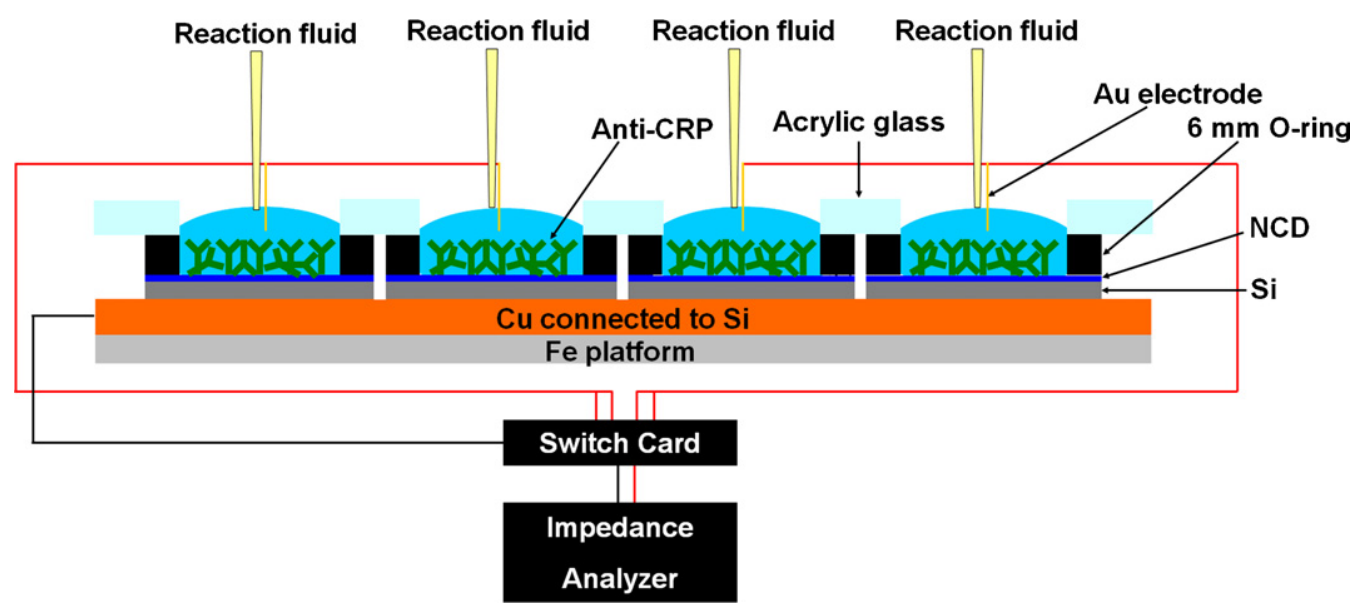

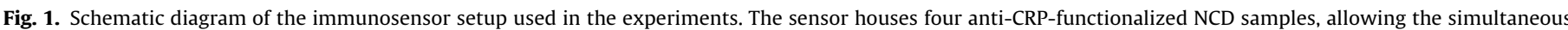
measurement of four conditions.

Adopted from [33].

into the wells of a microtiter plate. This microtiter plate was subsequently placed inside an ELISA reader, and the absorption of the colored product was measured at $405 \mathrm{~nm}$.

To examine the adsorption of CRP molecules and AP-labeled anti-CRP antibodies to unmodified NCD, two H-terminated samples that were not coated with anti-CRP but only blocked with $6 \%$ BSA were also treated with CRP and CRP buffer, respectively.

\subsection{Sensor setup}

The sensor setup devised for the development of a CRP-sensitive immunosensor is displayed in Fig. 1. Four separate NCD samples of $1 \mathrm{~cm}^{2}$ coated with anti-CRP antibodies, functioning as working electrodes, were mounted on a copper $(\mathrm{Cu})$ back contact using silver $(\mathrm{Ag})$ paste. Rubber O-rings with a diameter of $6 \mathrm{~mm}$ and an acrylic glass lid containing circular openings of equal size were pressed onto the samples to create four reaction wells above the NCD samples.

Each well was filled with $140 \mu$ l of reaction fluid. Au wires, placed $\sim 1 \mathrm{~mm}$ above each NCD surface and in contact with the reaction fluid, were used as counter-electrodes. $\mathrm{Ag} / \mathrm{AgCl}$ electrodes were also tested. However, noise was lowest using Au. Working and counter-electrodes were connected to the impedance analyzer with shielded cables. Hence, four signals were recorded simultaneously.

\subsection{Electrochemical Impedance Spectroscopy (EIS)}

EIS was performed using a Hewlett Packard 4194A Impedance/Gain-Phase Analyzer (Agilent, Diegem, Belgium). The impedance is measured by applying an AC potential $(U)$ of $10 \mathrm{mV}$ to the measurement cell. The response to this potential is an AC current signal $(I)$. The complex impedance $(Z)$ was measured for 50 frequencies, equidistant on a logarithmic scale, in a frequency range from $100 \mathrm{~Hz}$ to $1 \mathrm{MHz}$. The duration of one complete frequency sweep per channel was $8 \mathrm{~s}$. A Keithley 7001 switch card (Keithley Instruments, B.V., Sint-Pieters-Leeuw, Belgium) was used to switch between the four channels after each frequency sweep.

Real-time impedance curves from anti-CRP-functionalized or BSA-functionalized NCD samples mounted into the above described setup were recorded continuously during stabilization in buffer before antigen addition, antigen addition, and rinsing. The reaction wells were first filled with pure $(1 \times$ or $0.1 \times)$ PBS buffer, and the device was allowed to stabilize until the difference in $Z$ between two successive frequency sweeps was negligible throughout the entire frequency range.

For the specificity experiments, $72 \mathrm{pmol}(500 \mathrm{nM})$ of either CRP or plasminogen was then added to the PBS buffer, and allowed to react for $1 \mathrm{~h}$. Plasminogen $(81 \mathrm{kDa})$ is present in blood, making it a good competitor molecule. When converted into its active form plasmin, it plays an important role in wound healing. After the reaction, the reaction wells were rinsed and refilled with pure $(1 \times$ or $0.1 \times$ ) PBS buffer.

For the sensitivity analysis, $14.4,1.44$, and $0 \mathrm{pmol}(100,10,0 \mathrm{nM})$ of CRP, diluted in CRP buffer, was added to the PBS buffer. After the reaction, the reaction wells were rinsed and refilled with pure $(1 \times$ or $0.1 \times$ ) PBS buffer.

For the serum measurements, spiked and unspiked serum was added to pure serum to a final concentration of $72 \mathrm{pmol}(500 \mathrm{nM})$ of CRP. After the reaction, the reaction wells were rinsed and refilled with pure serum.

Experiments were performed at $37^{\circ} \mathrm{C}$, by placing the sensor setup inside a humidity-controlled oven, which also provided electromagnetic shielding.

\subsection{Statistical analysis}

The significance of the impedance behavior during serum measurements was determined using Student's $t$-test.

\section{Results and discussion}

\subsection{Reference ELISA procedure to verify the functionality of the adsorbed antibodies}

To verify the suitability of H-terminated NCD as a substrate for the physisorption of anti-CRP, as well as the functionality of these adsorbed antibodies, two H-terminated NCD samples were coated with $20 \mathrm{nM}$ unlabeled anti-CRP antibodies and 6\% BSA, as described above. To examine the non-specific adsorption of CRP and AP-labeled anti-CRP antibodies to diamond, two additional $\mathrm{H}$ terminated samples were only coated with $6 \%$ BSA. For both NCD couples (anti-CRP-coated and BSA-coated H-terminated NCD), one sample was treated with CRP, while the other was incubated with CRP buffer. The negative control samples (coated or uncoated with anti-CRP) treated with CRP buffer, provided insight in the nonspecific adsorption of AP-labeled anti-CRP antibodies to coated or uncoated NCD. The negative control sample that was not coated 


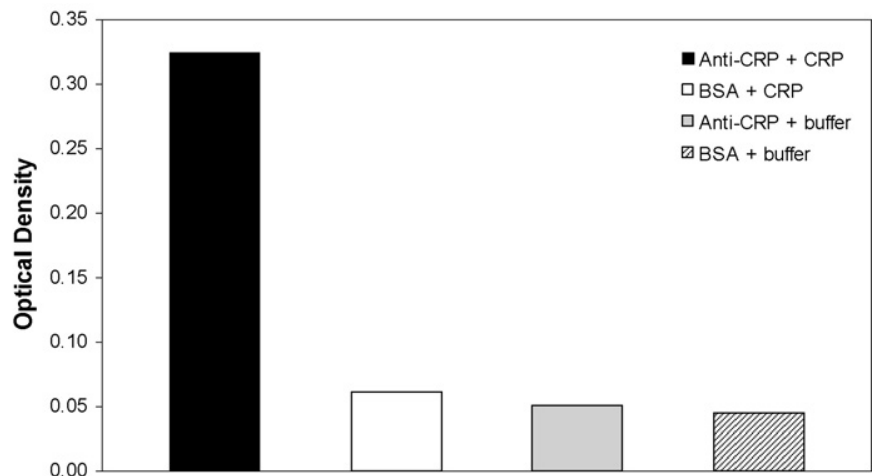

Fig. 2. Colorimetric measurements of differently functionalized NCD samples, displaying the efficiency of the antibody/antigen-complex formation and the amount of non-specific adsorption of CRP and AP-labeled anti-CRP.

with anti-CRP but was treated with CRP indicated the amount of non-specific CRP adsorption to NCD. Detection occurred with an AP-labeled anti-CRP antibody and an AP-based substrate reaction. The absorption of the colored product was determined at $405 \mathrm{~nm}$. It was clear that the absorption is the highest for the sample allowing the formation of the antibody/antigen-complex, with minimal contributions due to non-specific adsorption (Fig. 2). H-terminated NCD was therefore considered a suitable substrate for antibody adsorption, as the biological functionality of the antibodies are retained.

\subsection{Specificity of the immunosensor}

The specificity of the sensor was investigated by comparing the influence of $72 \mathrm{pmol}$ CRP with that of $72 \mathrm{pmol}$ plasminogen on the impedance behavior of anti-CRP functionalized NCD samples.

\subsubsection{Nyquist plots and real-time immunosensing}

Real-time immunosensing experiments were performed as follows. The wells were first filled with $140 \mu$ l of $1 \times$ PBS, after which the impedance signal was allowed to stabilize. At the time corresponding to $t=0$, CRP was added to the first two wells, while plasminogen was added to the third and fourth well, ensuring that all final antigen concentrations were $500 \mathrm{nM}$. After $80 \mathrm{~min}$, the four wells were rinsed three times with pure $1 \times$ PBS buffer. In order to check for the presence of free antibodies on the NCD surface that was still available for antigen recognition after this procedure, and also to investigate the reliability of the sensing method, the addition of proteins was repeated as before. Fig. $3 \mathrm{~A}$ illustrates a typical real-time behavior of the impedance at $100 \mathrm{~Hz}$ during such an experiment.

Note that the start of the first antigen treatment, immediately after the first antigen addition to the buffer $(t=0)$, can be regarded as an internal reference point, corresponding to a condition where none of the antibodies have recognized their antigen. This allows the events in each channel to be evaluated relative to this point. Hence, the impedance in each channel has been normalized to this internal reference point.

In all of the channels, the direct effect of protein addition is an immediate steep impedance increase of $~ 5 \%$. After $80 \mathrm{~min}$, the impedance has increased with $20 \%$ in the first well treated with CRP (open squares) and with $10 \%$ in the second well treated with CRP (open triangles), with respect to $t=0$. A small decrease of $\sim 2 \%$ with respect to $t=0$ after the initial impedance increase was observed in the wells where plasminogen was added (full symbols).

During the second antigen recognition phase, both CRP channels no longer show the clear increase in impedance after the initial rise
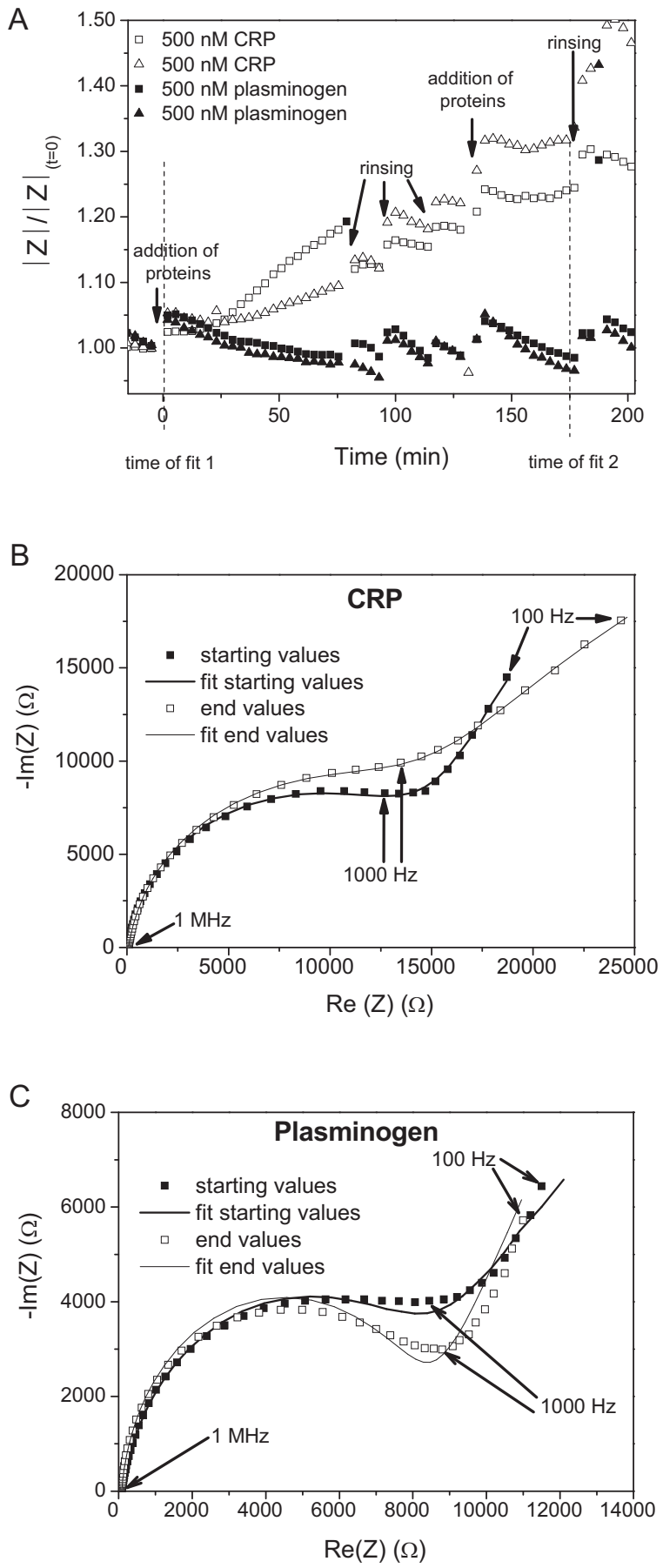

Fig. 3. Determination of the specificity of the CRP-directed immunosensor. (A) The real-time behavior of the impedance at $100 \mathrm{~Hz}$ during the total course of the experiment. The impedance values have been normalized to the value at the start of the first addition of proteins $(t=0)$. (B) Nyquist plot showing the impedance for the 50 frequencies at the start of the first CRP addition ( $\boldsymbol{\square})$ and at the end of the second CRP addition ( $\square$ ). (C) Nyquist plot showing the impedance for the 50 frequencies at the start of the first plasminogen addition ( $\boldsymbol{\square})$ and at the end of the second plasminogen addition ( $\square$ ). The full lines indicate fits to an equivalent circuit.

as observed during the first protein addition, indicating that most of the anti-CRP antibodies recognized their CRP targets during the first reaction. However, they have now reached an increase of more than $25 \%$ from the reference point $t=0$, possibly due to extensive ionic redistribution in the molecular layer during the rinsing steps. The channels where plasminogen was added show the same behavior during both additions. 


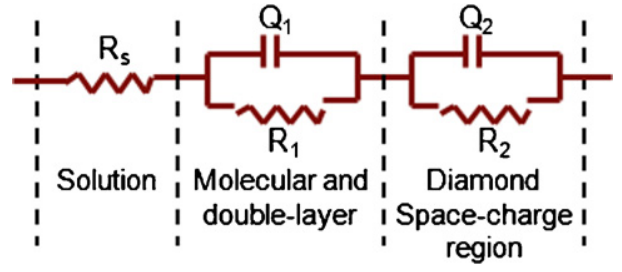

Fig. 4. The electrical circuit model used for fitting the impedance data.

Fig. 3B and $C$ shows the Nyquist plots of this experiment at the reference point $t=0$ (full squares) and at the end of the second protein addition (open squares) of one CRP- and one plasminogentreated channel, respectively. It is obvious that the impedance at low frequencies has increased during the CRP recognition (Fig. 3B). During plasminogen incubation (Fig. 3C), a completely different behavior is observed. The impedance has decreased over the frequency range from $100 \mathrm{~Hz}$ to $1000 \mathrm{~Hz}$. The results for the remaining channels are similar (data not shown).

\subsubsection{Fitting the results}

In order to get more insight in the physical meaning of the impedance variations, the Nyquist plots of Fig. 3B and C were analyzed using equivalent circuit models. This was done using the ZSimpWin software from Princeton Applied Research (MA, USA). The fits were performed over the total frequency range from $100 \mathrm{~Hz}$ to $1 \mathrm{MHz}$. For most of the data, good fits $\left(\chi^{2} \sim 4 \times 10^{-4}\right)$ could be obtained by using the model circuit given in Fig. 4 , and they are illustrated by the full lines in Fig. 3B and C.

The circuit can be divided into three components: (a) the solution resistance $R_{\mathrm{s}}$, between the Au electrode and the NCD surface, (b) a resistance $R_{Q_{1}}$ and a constant phase element $Q_{1}$ in parallel, corresponding to the molecular layer and its associated double-layer on the surface, (c) a resistance $R_{Q_{2}}$ and a constant phase element $Q_{2}$ in parallel, corresponding to the space-charge region in the NCD. The impedance of a $Q$ element is defined by $Z=\left[Q(i w)^{n}\right]^{-1}$. For our data, $n$ varied between 0.7 and 1 . A value of $n=1$ is equivalent to a 'perfect' capacitor. A value of $n=0.5$ corresponds to a Warburg impedance [34]. The parameter values obtained for the fits shown in Fig. 3B and $\mathrm{C}$ are indicated in Table 1.

From the fit results it can be seen that the increase of the impedance at low frequencies $(100-1000 \mathrm{~Hz})$ during CRPtreatment in Fig. 3B corresponds with a significant decrease of the $Q_{1}$ value with $42 \pm 7 \%$. This was consistently observed, and indicates a smaller capacity for the molecular layer after CRP recognition at the surface. The additional layer of CRP antigens on top of the anti-CRP antibodies increases the thickness of the molecular layer and changes its dielectric properties, decreasing its capacitance.

However, in the well where plasminogen was added, only the other parallel element $R_{2} Q_{2}$, reflecting the space-charge region of the NCD, shows significant changes. The constant phase element $Q_{2}$ decreases with $70 \pm 5 \%$. It should be mentioned that this large decrease of $Q_{2}$ was not consistently observed for all plasminogen additions. However, what was observed in each experiment for the reference channel (CRP buffer addition, plasminogen addition, BSA-coated NCD), is a slight decrease of the total impedance at low frequencies, as illustrated in the following section.

\subsection{Reliability of the immunosensor}

In order to get more insight in the reliability of the immunosensor, we performed a variation on the specificity measurements. The effect of antigen addition on H-terminated NCD samples functionalized with anti-CRP was compared with $\mathrm{H}$-terminated NCD coated
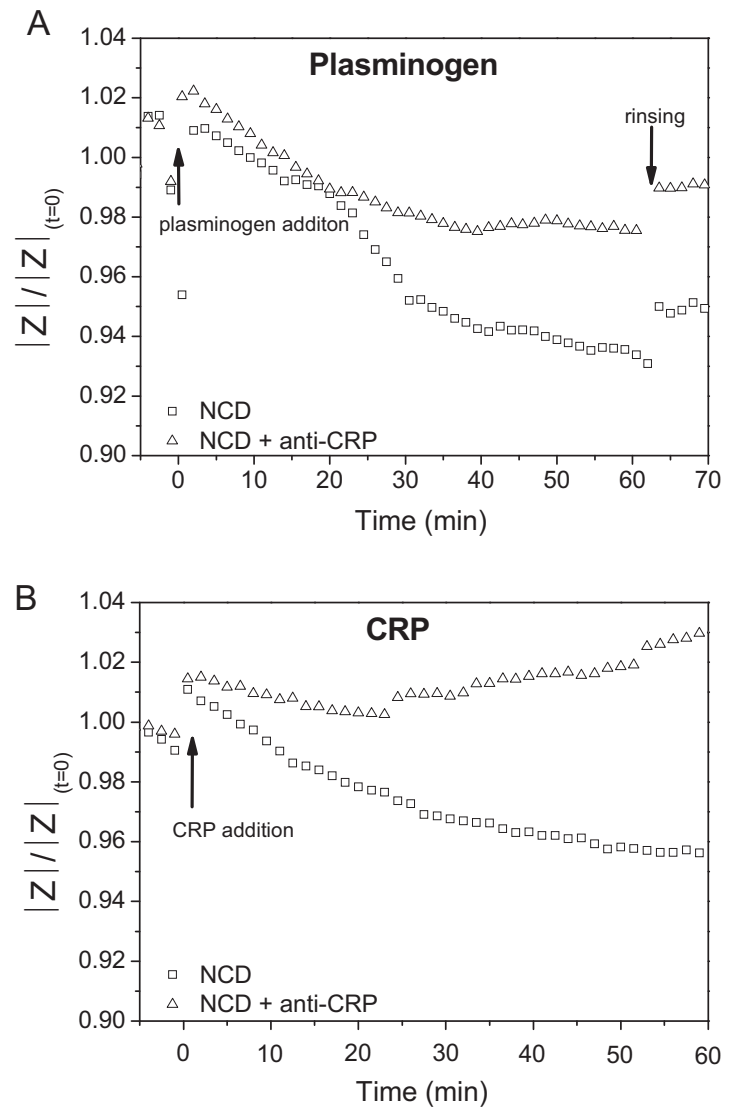

Fig. 5. Real-time impedance spectra taken at $100 \mathrm{~Hz}$. (A) The effect of $500 \mathrm{nM}$ plasminogen addition on bare NCD and NCD functionalized with anti-CRP. (B) The effect of $500 \mathrm{nM}$ CRP addition to the same samples after rinsing and stabilization.

with BSA. A typical result of such an experiment is illustrated in Fig. 5 where the normalized impedance at $100 \mathrm{~Hz}$ is given.

After stabilization, first plasminogen was added to the wells, so that the final concentration in the wells was $500 \mathrm{nM}$. The resulting effect is given in Fig. 5A. After the typical initial impedance increase, there was a decrease of $\sim 2$ and $6 \%$ with respect to $t=0$ for both NCD types, comparable to the effect observed in Fig. 3A.

In a second step, and after rinsing, CRP was added to these wells in a final concentration of $500 \mathrm{nM}$. As can be seen in Fig. 5B, CRP addition has the characteristic increasing effect only on the NCD sample functionalized with anti-CRP (open triangles), associated with a decrease in molecular layer capacitance. The level of this increase, however, is slightly smaller than in Fig. 3A.

\subsection{Sensitivity of the immunosensor}

A clear effect on the impedance is also reproducibly observed in real-time when exposing the anti-CRP-modified H-terminated NCD samples to different concentrations of CRP. Fig. 6A shows a typical result of experiments where $14.4 \mathrm{pmol}$ of CRP, $1.4 \mathrm{pmol}$ of CRP, and pure CRP buffer were added to $140 \mu$ l of $0.1 \times$ PBS buffer in wells 1,2 , and 3 , respectively. The final CRP concentrations in these three wells then corresponded to $100 \mathrm{nM}, 10 \mathrm{nM}$ and $0 \mathrm{nM}$, respectively. Real-time impedance spectra were taken at $100 \mathrm{~Hz}$.

The impedance at $100 \mathrm{~Hz}$ changes in a concentration-dependent manner. The different impedance spectra, corresponding to the different CRP concentrations, can be clearly distinguished from each other within $40 \mathrm{~min}$. The impedance of the reference channel (open circles) shows a characteristic decrease, while the impedance of the channel with a CRP concentration of $100 \mathrm{nM}$ (open squares) is 
Table 1

Results of fitting the impedance spectra at the start of the first and at the end of the second addition of CRP and plasminogen.

\begin{tabular}{|c|c|c|c|c|c|c|}
\hline \multirow[t]{2}{*}{ Element } & \multicolumn{3}{|l|}{ CRP } & \multicolumn{3}{|c|}{ Plasminogen } \\
\hline & Start & End & Significant effect (\%) & Start & End & Significant effect (\%) \\
\hline$R_{\mathrm{S}}(\Omega)$ & $69.7 \pm 1.3^{\mathrm{a}}$ & $66.2 \pm 0.8$ & $-5 \pm 2$ & $74.1 \pm 1.9$ & $81 \pm 4$ & $+9 \pm 6$ \\
\hline$Q_{1}\left(\mu S s^{n}\right)$ & $0.48 \pm 0.05$ & $0.279 \pm 0.015$ & $-42 \pm 7$ & $1.3 \pm 0.3$ & $1.7 \pm 0.7$ & - \\
\hline$n$ & $0.77 \pm 0.02$ & $0.78 \pm 0.01$ & - & $0.71 \pm 0.03$ & $0.69 \pm 0.06$ & - \\
\hline$R_{1}(\Omega)$ & und. $^{\mathrm{b}}$ & und. & - & und. & und. & - \\
\hline $\mathrm{Q}_{2}\left(\mathrm{nS} \mathrm{s}^{n}\right)$ & $12.1 \pm 0.9$ & $13.3 \pm 0.7$ & - & $21.2 \pm 1.9$ & $6.2 \pm 0.9$ & $-70 \pm 5$ \\
\hline$n$ & $0.96 \pm 0.01$ & $0.95 \pm 0.01$ & - & $0.89 \pm 0.01$ & $0.96 \pm 0.01$ & $+7 \pm 2$ \\
\hline$R_{2}(\mathrm{k} \Omega)$ & $1.31 \pm 0.04$ & $1.28 \pm 0.04$ & - & $7.8 \pm 0.4$ & $7.8 \pm 0.3$ & - \\
\hline
\end{tabular}

a Reported uncertainties are standard deviations as obtained from the data fits.

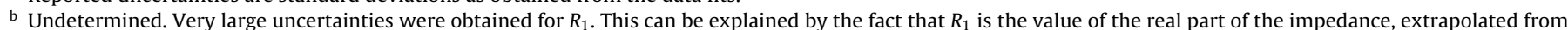

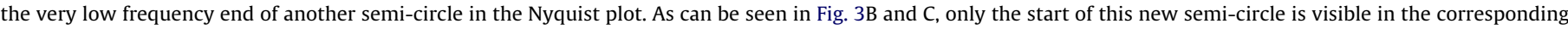
Nyquist plots, leading to a large uncertainty in the fit results of $R_{1}$.

clearly increasing, and shows a comparable behavior to the effect described in Figs. 3A and 5B, associated with a decrease in molecular layer capacitance. Like in Fig. 5B, the level of this increase, however, is again smaller than in Fig. 3A. The impedance of the channel with the lowest CRP concentration (open triangles), how-

A

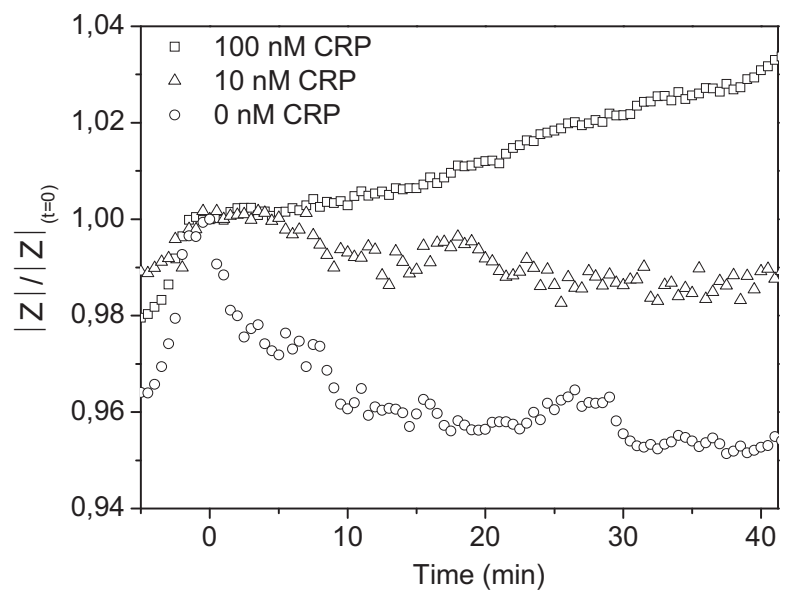

B

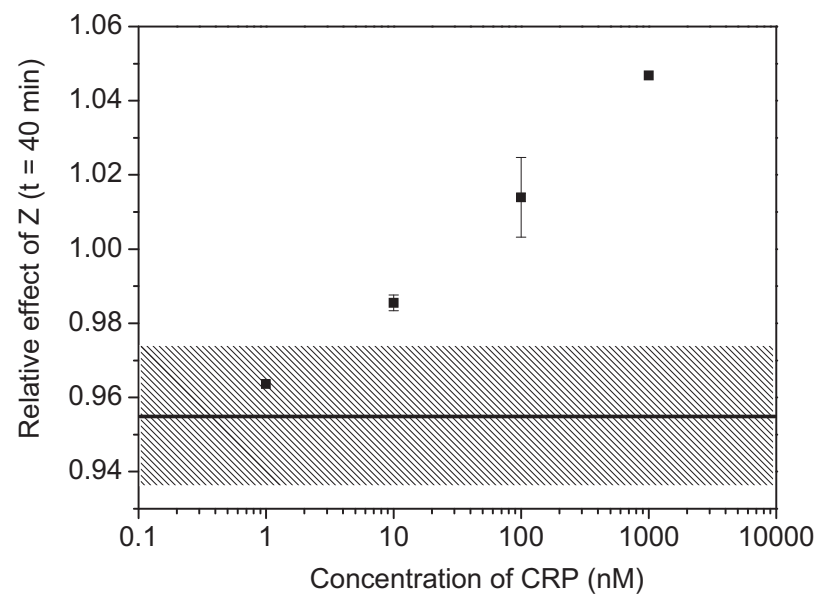

Fig. 6. Concentration-dependent sensitivity of the sensor for CRP. (A) Real-time impedance spectra at $100 \mathrm{~Hz}$ of different concentrations of CRP. (B) Calibration plot representing the relative effect on $Z$ after $40 \mathrm{~min}$ for different concentrations of CRP. The black line shows the relative effect on $Z$ for $0 \mathrm{nM}$ of CRP (pure CRP buffer) and the gray box delineates the standard deviation. The data are generated from 3 independent measurements. ever, already starts to show a slight decrease, indicating a lower density of antigen/antibody-complexes, and approaching the condition of non-recognition, also seen with buffer and plasminogen treatment. Fig. 6B shows a calibration for concentrations varying from $1 \mathrm{nM}$ (or $\sim 1.25 \mu \mathrm{g} \mathrm{ml}^{-1}$ ) to $1000 \mathrm{nM}$. The data represent the mean value and standard deviation for the change in $Z$ after $40 \mathrm{~min}$ of CRP treatment. The mean value and standard deviation for pure CRP buffer ( $0 \mathrm{nM} \mathrm{CRP})$ is indicated by the full line and shaded area, respectively. From this curve it is clear that $10 \mathrm{nM}$, was the lowest concentration that could reproducibly be discriminated from buffer. This limit of detection was comparable to the work described by Hu et al. [8], who could detect $8 \mathrm{nM}\left(1 \mu \mathrm{g} \mathrm{ml}^{-1}\right)$ of purified CRP, and even slightly better than the work described by Meyer et al. [9], who reached a detection limit of $16-40 \mathrm{nM}\left(2-5 \mu \mathrm{g} \mathrm{ml}^{-1}\right)$ of purified CRP. All of these studies were done in real-time, with results of our group and Hu et al. [8] being obtained in the minute-scale, while an experiment of Meyer et al. [9] takes about $3 \mathrm{~h}$. However, further improvements are needed to reach and exceed that of the hs-CRP assay and that of Meyer et al. [10] in crude samples. However, these are endpoint assays.

The real-time impedance spectra observed at $100 \mathrm{~Hz}$ (Figs. 3A, 5 and 6A) indicate that, when comparing the sample channel to a reference channel in the same measurement, a good discrimination between the two conditions is obtained after 40 min of reaction. However, because of the good reproducibility of the sensor, it is also possible to compare the sample channels of separate measurements. This is illustrated in Fig. 7, where the CRP-dependent impedance curve (black squares) is the average of 5 independent impedance measurements at $100 \mathrm{~Hz}$ on anti-CRP

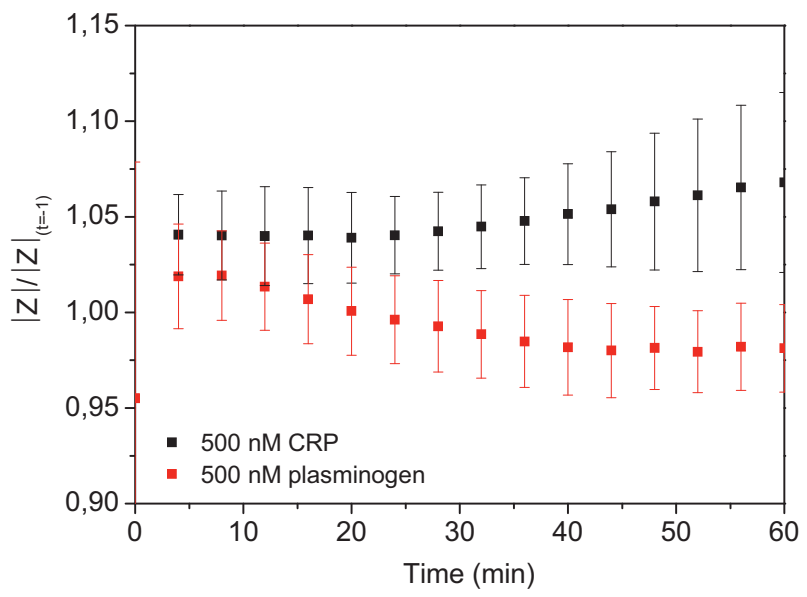

Fig. 7. An averaging of impedance spectra taken at $100 \mathrm{~Hz}$ during $500 \mathrm{nM}$ CRP addition $(\square)$ and $500 \mathrm{nM}$ plasminogen addition $(\square)$. 


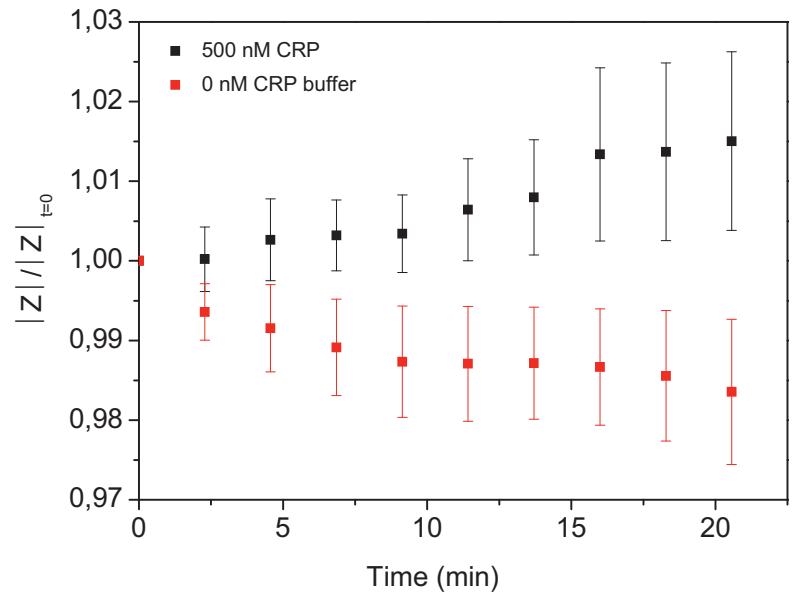

Fig. 8. An averaging of 6 impedance spectra taken at $100 \mathrm{~Hz}$ during the addition of spiked serum (black) to reach a final CRP concentration of $500 \mathrm{nM}$, and unspiked serum ( $\square)$.

functionalized samples, while the non-specific plasminogendependent impedance curve (red squares) is the average of 7 independent impedance measurements at $100 \mathrm{~Hz}$ on anti-CRP functionalized samples. The added concentrations were identical to the previous experiments. The error bars indicate standard deviations. It can be seen that, after $25 \mathrm{~min}$, the two types of curves significantly diverge, and obtain a range of values specific for reference and sample. The rise in impedance remains consistently characteristic for the specific CRP recognition.

\subsection{Serum}

To investigate the immunosensor's suitability to detect CRP in a complex and clinically relevant matrix, the reaction wells were first filled with $140 \mu \mathrm{l}$ of pure human serum. After stabilization of the impedance signal, spiked serum was added so that the final CRP concentration was $500 \mathrm{nM}(t=0)$. For the negative control, the same volume of unspiked human serum was added. Fig. 8 shows the average of 6 independent impedance measurements at $100 \mathrm{~Hz}$ on anti-CRP functionalized samples in human serum.

The negative control shows the typical decrease in impedance signal. The spiked serum causes a characteristic impedance increase of $1.6 \%$ after $30 \mathrm{~min}$. As compared to the measurements in buffer, shown in Fig. 7, the impedance effect is slightly less, probably due to the high complexity of the medium. However, difference in impedance behavior between spiked and unspiked serum was significant $(p>0.045)$, indicating that real-time and label-free detection of CRP is possible in human serum.

It should be remarked that, in comparison to our impedimetric experiments on DNA-functionalized NCD samples [32], the stabilization of the immunosensor impedance signal after the disturbance of the system by pipetting manipulations takes longer. This increased stabilization time can be attributed to the fact that the DNA probes were covalently bound to the NCD through fatty acid linker molecules, whereas the antibodies were physically adsorbed to H-terminated NCD. Some degree of gradual dissociation and re-adsorption of the antibodies from and to the surface can therefore not be excluded, resulting in a dynamic molecular layer. It has been reported that covalently attaching the receptor molecules to a surface significantly decreases signal drift as compared to physically adsorbed receptor molecules [35].

\section{Conclusions}

In this work, the potential of EIS on H-terminated NCD is demonstrated for the development of a real-time and labelfree immunosensor. The suitability of this diamond substrate for functional antibody adsorption was demonstrated with an ELISA reference technique. In this study a specific discrimination between CRP and plasminogen was obtained in real-time at low frequencies $(100 \mathrm{~Hz})$

Specific CRP recognition by the physisorbed anti-CRP antibodies was consistently associated with an increase in impedance, due to a decreased capacitance of the molecular layer caused by CRP binding. The level of increase varied between 5 and $20 \%$. On the other hand, non-specific conditions, either by the addition of plasminogen or buffer to anti-CRP functionalized NCD or the use of BSA-coated NCD, were characterised by a small impedance decrease at $100 \mathrm{~Hz}$ of $\sim 5 \%$.

The sensitivity of the immunosensor was analyzed by incubating the NCD samples with different concentrations of CRP. At $100 \mathrm{~Hz}$, the lowest concentration of CRP used in our experiments (10 nM) was still clearly distinguishable from the reference channel treated with buffer within $20 \mathrm{~min}$. This indicates the possibility that our prototype immunosensor for CRP can reach sensitivity within the physiologically relevant concentration range, important to discriminate between healthy controls $(8-10 \mathrm{nM})$ and patients at risk for CVD (>10 nM).

Preliminary serum measurements were also performed, and showed that the impedimetric immunosensor was capable of significantly distinguishing between spiked and unspiked serum. The applicability of the sensor in a real biological matrix displays genuine clinical relevance, and will allow for its use as a point-of-care diagnostic device.

For future experiments, a covalent attachment between antibody and NCD is recommended to decrease the stabilization time of the impedance signal.

Another point of improvement is the miniaturization of the sensor setup. This will likely result in a decrease of the reaction time through its effects on diffusion and double-layer disturbances. First of all, decreasing the reaction volume will significantly decrease the diffusion time of the antigens to the surface. Secondly, this smaller reaction volume will likely shorten the relaxation time of the double-layer after disturbances.

Furthermore, the analysis of frequencies below $100 \mathrm{~Hz}$, which will be even more sensitive to events in the molecular layer, will increase the reproducibility of the magnitude of the impedance effects during recognition of different concentrations of CRP.

\section{Acknowledgements}

The research was performed in the framework of the IAP VI program 'Quantum Effects in Clusters and Nanowires', the Scientific Research Community WOG WO.035.04N 'Hybrid Systems at Nanometer Scale', the Fund for Scientific Research (FWO) projects G.082909 'Synthetic diamond films as platform material for novel DNA sensors with electronic detection principles' and G.0068.07 "Growth, characterisation and simulation of nanocrystalline and ultrananocrystalline PE-CVD diamond films', and the Methusalem project 'NANO Antwerp-Hasselt'. Lars Grieten and Veronique Vermeeren are funded by IWT. We also gratefully acknowledge impulse financing by the transnational University Limburg tUL. Technical assistance by J. Baccus, J. Soogen, and B. Conings in the development of the measurement setups is greatly appreciated.

\section{References}

[1] V. Pasceri, J.T. Willerson, E.T. Yeh, Direct proinflammatory effect of C-reactive protein on human endothelial cells, Circulation 102 (2000) 2165-2168. 
[2] F. Haverkate, S.G. Thompson, S.D. Pyke, J.R. Gallimore, M.B. Pepys, European Concerted Action on Thrombosis and Disabilities Angina Pectoris Study Group Production of C-reactive protein and risk of coronary events in stable and unstable angina, Lancet 349 (1997) 462-466.

[3] H.D. Sesso, J.E. Buring, N. Rifai, G.J. Blake, J.M. Gaziano, P.M. Ridker, C-reactive protein and the risk of developing hypertension, JAMA 290 (2003) 2945-2951.

[4] P.M. Ridker, M. Cushman, M.J. Stampfer, R.P. Tracy, C.H. Hennekens, Inflammation, aspirin, and the risk of cardiovascular disease in apparently healthy men, N. Engl. J. Med. 336 (1997) 973-979.

[5] D. Lloyd-Jones, R. Adams, M. Carnethon, G. De Simone, T.B. Ferguson, K. Flegal, E. Ford, K. Furie, A. Go, K. Greenlund, N. Haase, S. Hailpern, M. Ho, V. Howard, B. Kissela, S. Kittner, D. Lackland, L. Lisabeth, A. Marelli, M. McDermott, J. Meigs, D. Mozaffarian, G. Nichol, C. O’Donnell, V. Roger, W. Rosamond, R. Sacco, P. Sorlie, R. Stafford, J. Steinberger, T. Thom, S. Wasserthiel-Smoller, N. Wong, J. Wylie-Rosett, Y. Hong, Heart disease and stroke statistics - 2009 update: a report from the American Heart Association Statistics Committee and Stroke Statistics Subcommittee, Circulation 119 (2009) 480-486.

[6] World Health Organization, World Health Statistics Annual, Report, 1998

[7] Y. Hokama, R.M. Nakamura, C-reactive protein: current status and future perspectives, J. Clin. Lab. Anal. 1 (1987) 15-27.

[8] W.P. Hu, H.Y. Hsu, A. Chiou, K.Y. Tseng, H.Y. Lin, G.L. Chang, S.J. Chen, Immunodetection of pentamer and modified C-reactive protein using surface plasmon resonance biosensing, Biosens. Bioelectron. 21 (2006) 1631-1637.

[9] M.H. Meyer, M. Hartmann, M. Keusgen, SPR-based immunosensor for the CRP detection-a new method to detect a well known protein. Biosens. Bioelectron. 21 (2006) 1987-1990.

[10] M.H. Meyer, M. Hartmann, H.J. Krause, G. Blankenstein, B. Mueller-Chorus, J. Oster, P. Miethe, M. Keusgen, CRP determination based on a novel magnetic biosensor, Biosens. Bioelectron. 22 (2007) 973-979.

11] P. Cooreman, R. Thoelen, J. Manca, M. vandeVen, V. Vermeeren, L. Michiels, M Ameloot, P. Wagner, Impedimetric immunosensors based on the conjugated polymer PPV, Biosens. Bioelectron. 20 (2005) 2151-2156.

[12] R.E. Ionescu, N. Jaffrezic-Renault, L. Bouffier, C. Gondran, S. Cosnier, D.G Pinacho, M.P. Marco, F.J. Sanchez-Baeza, T. Healy, C. Martelet, Impedimetric immunosensor for the specific label free detection of ciprofloxacin antibiotic, Biosens. Bioelectron. 23 (2007) 549-555.

[13] W. Yang, J.E. Butler, J.N. Russell Jr., R.J. Hamers, Direct electrical detection of antigen-antibody binding on diamond and silicon substrates using Electrical Impedance Spectroscopy, Analyst 132 (2007) 296-306.

[14] G.M. Swain, M. Ramesham, The electrochemical activity of boron-doped polycrystalline diamond thin-film electrodes, Anal. Chem. 65 (1993) 345-351.

[15] J.E. Field, Mechanical and physical properties of diamond, Inst. Phys. Conf. Ser. 75 (1987) 181-205.

[16] S. Yamanaka, D. Takeuchi, H. Watanabe, H. Okushi, K. Kajimura, Lowcompensated boron-doped homoepitaxial diamond films, Diamond Relat. Mater. 9 (2000) 956-959.

[17] J. Pernot, P.N. Volpe, F. Omnes, P. Muret, V. Mortet, K. Haenen, T. Teraji, Hall hole mobility in boron-doped homoepitaxial diamond, Phys. Rev. B 81 (2010).

18] K. Haenen, A. Lazea, J. Barjon, J. D'Haen, N. Habka, T. Teraji, S. Koizumi, V. Mortet, P-doped diamond grown on (1 110$)$-textured microcrystalline diamond: growth, characterization and devices, J. Phys.: Condens. Matter 21 (2009).

[19] B Rezek, J.A. Garrido, M. Stutzmann, C.E. Nebel, E. Snidero, P. Bergonzo, Loca oxidation of hydrogenated diamond surfaces for device fabrication, Phys. Status Solidi A 193 (2002) 523-528.

[20] Y. Sasaki, H. Kawarada, Low drift and small hysteresis characteristics of diamond electrolyte-solution-gate FET, J. Phys. D: Appl. Phys. 43 (2010).

[21] L. Tang, C. Tsai, W.W. Gerberich, L. Kruckeberg, D.R. Kania, Biocompatibility of chemical-vapour-deposited diamond, Biomaterials 16 (1995) 483-488.

[22] K. Takahashi, M. Tanga, O. Takai, H. Okamura, DNA bonding to diamond, Biol. Ind. 17 (2000) 44-51.

[23] K. Ushizawa, Y. Sato, T. Mitsumori, T. Machinami, T. Ueda, T. Ando, Covalen immobilization of DNA on diamond and its verification by diffuse reflectance infrared spectroscopy, Chem. Phys. Lett. 351 (2002) 105-108.

[24] J. Wang, M.A. Firestone, O. Auciello, J.A. Carlisle, Functionalization of ultrananocrystalline diamond films by electrochemical reduction of aryldiazonium salts, Langmuir 20 (2004) 450-456.

25] D. Shin, B. Rezek, D. Tokuda, D. Takeuchi, H. Watanabe, T. Nakamura T. Yamamoto, C.E. Nebel, Photo- and electrochemical bonding of DNA to single crystalline CVD diamond, Phys. Status Solidi A 203 (2006) 3245-3272.

[26] H. Uetsuka, D. Shin, N. Tokuda, K. Saeki, C.E. Nebel, Electrochemical grafting of boron-doped single-crystalline chemical vapor deposition diamond with nitrophenyl molecules, Langmuir 23 (2007) 3466-3472.

[27] P. Christiaens, V. Vermeeren, S. Wenmackers, $M$. Daenen, K. Haenen, M. Nesladek, M. vandeVen, M. Ameloot, L. Michiels, P. Wagner, EDC-mediated DNA attachment to nanocrystalline CVD diamond films, Biosens. Bioelectron. 22 (2006) 170-177.

[28] V. Vermeeren, S. Wenmackers, M. Daenen, K. Haenen, O.A. Williams, M. Ameloot, V.M. Vande, P. Wagner, L. Michiels, Topographical and functional characterization of the ssDNA probe layer generated through EDCmediated covalent attachment to nanocrystalline diamond using fluorescence microscopy, Langmuir 24 (2008) 9125-9134

[29] W. Yang, O. Auciello, J.E. Butler, W. Cai, J.A. Carlisle, J.E. Gerbi, D.M. Gruen, T. Knickerbocker, T.L. Lasseter, J.N. Russell Jr., L.M. Smith, R.J. Hamers, DNAmodified nanocrystalline diamond thin-films as stable, biologically active substrates, Nat. Mater. 1 (2002) 253-257.
[30] A. Härtl, E. Schmich, J.A. Garrido, J. Hernando, S.C.R. Catharino, S. Walter, P. Feulner, A. Kromka, D. Steinmuller, M. Stutzmann, Protein-modified nanocrystalline diamond thin films for biosensor applications, Nat. Mater. 3 (2004) 736-742.

[31] S. Wenmackers, V. Vermeeren, M. vandeVen, M. Ameloot, N. Bijnens, K. Haenen, L. Michiels, P. Wagner, Diamond-based DNA sensors: surface functionalization and read-out strategies, Phys. Status Solidi A 206 (2009) 391-408.

[32] V. Vermeeren, N. Bijnens, S. Wenmackers, M. Daenen, K. Haenen, O.A Williams, M. Ameloot, M. vandeVen, P. Wagner, L. Michiels, Towards a real-time, label-free, diamond-based DNA sensor, Langmuir 23 (2007) 13193-13202.

[33] N Bijnens, V. Vermeeren, M. Daenen, L. Grieten, K. Haenen, S. Wenmackers, O.A. Williams, M. Ameloot, M. VandeVen, L. Michiels, P. Wagner, Synthetic diamond films as a platform material for label-free protein sensors, Phys. Status Solidi A 206 (2009) 520-526.

[34] S. Grimnes, O.S. Martinsen, Bioimpedance and Bioelectricity, Academic Press, London, 2000.

[35] S. Carrara, V.K. Bhalla, C. Stagni, L. Benini, B. Riccò, B. Samorì, Improving probe immobilization for label-free capacitive detection of DNA hybridization on microfabricated gold electrodes, Sens. Transducers 88 (2008) 31-39.

\section{Biographies}

Veronique Vermeeren obtained her $\mathrm{PhD}$ in 2008 at Hasselt University (Belgium), where she developed electronic nanocrystalline diamond-based biosensors, more specifically DNA and immunosensors. In 2008, she received an IWT postdoctoral grant for two years to continue her research on diamond-based biosensors. Since October 2010, she is employed at the Biomedical Research Institute (BIOMED) of Hasselt University, where she coordinates several research projects.

Lars Grieten graduated from Hasselt University (Belgium) in 2007 with a master's degree in Bioelectronics and Nanotechnology. In that year, he also started his PhD at the Institute for Material Research (IMO) of Hasselt University. He is currently working on the development of nanocrystalline diamond-based biosensor architectures for the label-free detection of proteins.

Natalie Vanden Bon obtained a bachelor's degree in Chemistry at Hasselt University (Belgium) in 2007. After her bachelor's degree she received a master's degree in Biochemistry and Biotechnology at the University of Antwerp in 2009. In September of that year, she started her PhD at the Biomedical Research Institute (BIOMED) of Hasselt University focusing on the development of nanocrystalline diamond-based biosensors, more specifically DNA and immunosensors.

Nathalie Bijnens obtained her PhD in 1998 at the Catholic University Leuven (Belgium), where she studied nuclear spectroscopy. She was involved in several experiments at different nuclear facilities (Darmstadt, Geneva, Chicago and Louvainla-Neuve). From 2000 until 2003 she worked in the development department of Philips Lighting. After two years of maternity leave, she was a postdoctoral researcher in materials science at the Institute for Material Research (IMO) of Hasselt University (Belgium). In close cooperation with the Biomedical Research Institute (BIOMED) of Hasselt University, she performed experiments on label-free DNA and protein sensors. At present, Nathalie Bijnens is staff member of education and research in the field of cardiovascular biomechanics at the Eindhoven University of Technology (The Netherlands).

Sylvia Wenmackers received a PhD in Physics in 2008 at Hasselt University (Belgium). She was affiliated to the Biosensor-group of the Institute for Materials Research (IMO), where she worked on the characterisation of biosensor surfaces, in particular DNA-sensors based on nanocrystalline diamond. To this end, she employed optical methods such as confocal fluorescence microscopy and spectroscopic ellipsometry, as well as scanning probe and electronic techniques. In 2008 she received a post-doctoral fellowship of the Flemish Research Fund for Scientific Research (FWO - Vlaanderen)

Stoffel Janssens graduated from Vrije Universiteit Brussel (Belgium) in 2006 with a master's degree in Chemistry. $\mathrm{He}$ is currently working as a PhD student at the Institute for Material Research (IMO) of Hasselt University (Belgium), on the development and the characterisation of ultra-thin boron-doped nanocrystalline microwave enhanced chemical vapour deposited diamond films.

Ken Haenen obtained his bachelor in physics at the Hasselt University (Belgium) (then called Limburgs Universitair Centrum) in 1995, followed by a master's in Physics from the Katholieke Universiteit Leuven (Belgium), in 1997. In the same year, he started to pursue a $\mathrm{PhD}$, focusing his research on the characterisation of thin phosphorus doped CVD diamond films. The samples, which were considered to be the first diamond films in which active n-type doping was proven, were obtained through collaboration with NIRIM, Japan, now called NIMS, and analyzed using highly sensitive photocurrent-based spectroscopy techniques. After obtaining his doctoral degree from Hasselt University (2002), in 2004 he became group leader of the Wide Band Gap Materials (WBGM) research group of the Institute for Materials Research (IMO) from the same University, after spending a short postdoctoral period at NIMS. In 2008 he was appointed professor in experimental physics with focus on the deposition, characterisation and use of materials including CVD diamond, AIN, and $\mathrm{BN}$. 
Patrick Wagner obtained his $\mathrm{PhD}$ in 1994 at Technical University Darmstadt (Germany) in experimental solid state physics with a focus on cuprate superconductors. From 1995 until 2001, he was postdoctoral researcher in the Laboratory of Solid State Physics and Magnetism at Catholic University Leuven (Belgium), where he studied the magneto-transport properties of mixed-valency magnetic oxides. Since 2001, he is a professor of physics at Hasselt University (Belgium) and responsible for the development of label-free readout techniques for DNA-, protein-, and smallmolecule sensors. Patrick Wagner is recipient of a WE Heraeus award, a Marie-Curie Fellowship of the European Union, a Methusalem Grant of the Flemish Government and is past president of the Belgian Physical Society.
Luc Michiels is professor at Hasselt University (Belgium). Since 1999 he has been a member of the Biomedical Research Institute (BIOMED) and a member of the board of directors of BIOMED direction. In 2002, he started a multidisciplinary research project with the Institute of Materials Research (IMO). Within this group, he developed a universal prototype of biosensors that can be applied in the study of DNA hybridization and denaturation events in real-time reaching SNP sensitivity, and in the study of immunosensing using immunoglobulins as receptors. Within BIOMED, he is responsible for the Biosensor Development key research domain. 